Article

\title{
TrmL and TusA Are Necessary for rpoS and MiaA Is Required for $h f q$ Expression in Escherichia coli
}

\author{
Joseph I. Aubee ${ }^{1}$, Morenike Olu ${ }^{1,2}$ and Karl M. Thompson ${ }^{1, *}$ \\ 1 Department of Microbiology, College of Medicine, Howard University, Washington, DC 20059, USA; \\ joseph.aubee@howard.edu (J.I.A.); morenike.olu@bison.howard.edu (M.O.) \\ 2 Department of Biology, College of Arts and Sciences, Howard University, Washington, DC 20059, USA \\ * Correspondence: karl.thompson@howard.edu
}

Academic Editor: Valérie de Crécy-Lagard

Received: 1 January 2017; Accepted: 12 April 2017; Published: 4 May 2017

\begin{abstract}
Previous work demonstrated that efficient RNA Polymerase sigma S-subunit (RpoS) translation requires the N6-isopentenyladenosine i6A37 transfer RNA (tRNA) modification for UUX-Leu decoding. Here we investigate the effect of two additional tRNA modification systems on RpoS translation; the analysis was also extended to another High UUX-leucine codon (HULC) protein, Host Factor for phage $\mathrm{Q} \beta$ (Hfq). One tRNA modification, the addition of the 2'-O-methylcytidine/ uridine $34(\mathrm{C} / \mathrm{U} 34 \mathrm{~m})$ tRNA modification by tRNA (cytidine/uridine-2'O)-ribose methyltransferase $\mathrm{L}$ (TrmL), requires the presence of the $N^{6}$-isopentenyladenosine $37\left(\mathrm{i}^{6} \mathrm{~A} 37\right)$ and therefore it seemed possible that the defect in RpoS translation in the absence of $i^{6} \mathrm{~A} 37$ prenyl transferase (MiaA) was in fact due to the inability to add the C/U34m modification to UUX-Leu tRNAs. The second modification, addition of 2-thiouridine $\left(\mathrm{s}^{2} \mathrm{U}\right)$, part of $\left(\mathrm{mnm}^{5} \mathrm{~s}^{2} \mathrm{U} 34\right)$, is dependent on tRNA 2-thiouridine synthesizing protein A (TusA), previously shown to affect RpoS levels. We compared expression of $\mathrm{P}_{B A D^{-}-r p o S 990-l a c Z}$ translational fusions carrying wild-type UUX leucine codons with derivatives in which UUX codons were changed to CUX codons, in the presence and absence of TrmL or TusA. The absence of these proteins, and therefore presumably the modifications they catalyze, both abolished $\mathrm{P}_{B A D}$-rpoS990-lacZ translation activity. UUX-Leu to CUX-Leu codon mutations in $r p o S$ suppressed the trmL requirement for $P_{B A D}$-rpoS990-lac $Z$ expression. Thus, it is likely that the $\mathrm{C} / \mathrm{U} 34 \mathrm{~m}$ and $\mathrm{s}^{2} \mathrm{U} 34$ tRNA modifications are necessary for full rpoS translation. We also measured $\mathrm{P}_{B A D^{-} \text {-hf }} 306$-lacZ translational fusion activity in the absence of $\mathrm{C} / \mathrm{U} 34 \mathrm{~m}$ (trmL) or i ${ }^{6} \mathrm{~A} 37$ (miaA). The absence of $\mathrm{i}^{6} \mathrm{~A} 37$ resulted in decreased $\mathrm{P}_{B A D}$-hfq306-lac $Z$ expression, consistent with a role for $\mathrm{i}^{6} \mathrm{~A} 37$ tRNA modification for $h f q$ translation.
\end{abstract}

Keywords: $\mathrm{s}^{2} \mathrm{U} 34$ tRNA modification; C/U34m tRNA modification; $\mathrm{i}^{6} \mathrm{~A} 37$ tRNA modification; RpoS; Hfq; MiaA; TrmL; TusA; leucine; codon bias

\section{Introduction}

Escherichia coli $\operatorname{RpoS}\left(\sigma^{S}\right)$ is an alternative sigma factor that facilitates physiological adaptation to general starvation and stationary phase growth [1,2]. RpoS levels are tightly regulated, particularly at the post-transcriptional level. Several small regulatory RNAs modulate translational initiation in response to various environmental conditions [3-8]. In addition, RpoS stability is decreased by the activity of the ATP-dependent Protease ClpXP with the assistance of the Regulator of sigma S B (RssB) adaptor protein [9]. Conversely, RpoS is stabilized by three anti-adaptor proteins: Inhibitor of RssB activity in response to phosphate starvation (IraP), Inhibitor of RssB activity in response to magnesium starvation (IraM), and Inhibitor of RssB activity in response to DNA damage (IraD) [10-13].

RpoS expression is also regulated at the level of translation within the open reading frame (ORF). SsrA (tmRNA) is a small RNA that enters the A-site of stalled ribosomes and is translated into a 
C-terminal tag that targets truncated proteins for degradation by several cellular proteases [14]. SsrA is necessary for full RpoS translation [14]. Translation of RpoS is significantly affected by at least one tRNA modification, N6-isopentyl adenosine $\left(i^{6} \mathrm{~A}\right)$ [15]. tRNA dimethylallyltransferase (MiaA) catalyzes the prenylation $\left(\mathrm{i}^{6}\right)$ of adenine 37 (A37) to tRNAs that read UXX codons [16-18]. The mechanism of action of the $i^{6} \mathrm{~A} 37 \mathrm{tRNA}$ modification on RpoS expression is, at least in part, promotion of efficient UUX-leucine decoding (Figure 1) [19]. Here, we examined whether two other tRNA modification systems played roles in RpoS translation.

A

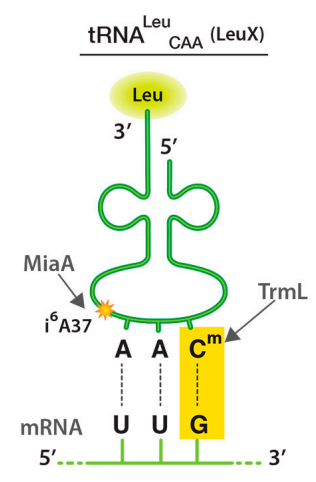

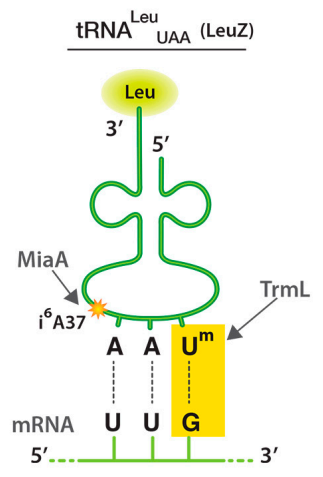

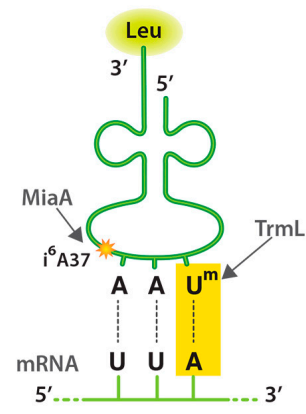

B
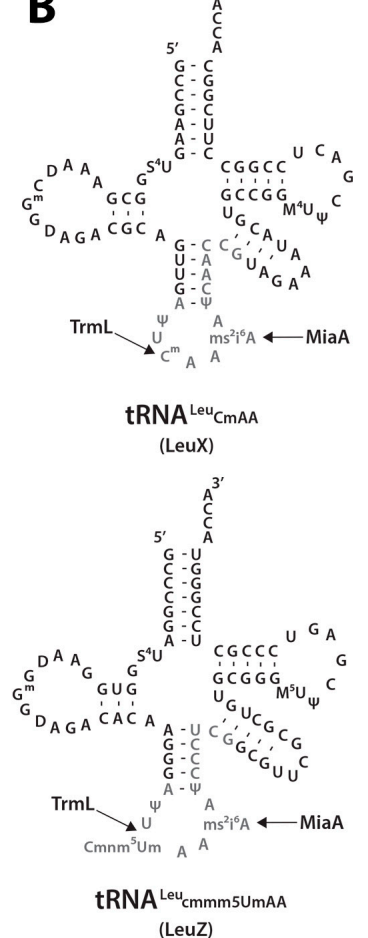

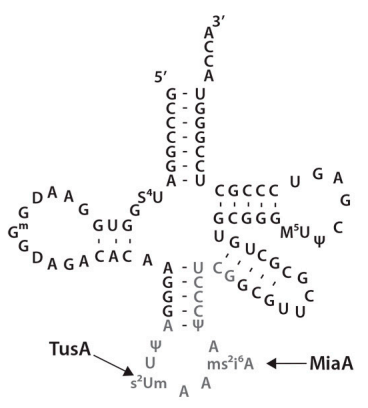

Figure 1. tRNA (cytidine/uridine-2'O)-ribose methyltransferase $L$ (TrmL), tRNA dimethylallyltransferase (MiaA), and tRNA 2-thiouridine synthesizing protein A (TusA) tRNA modifications and UUX-Leu codon recognition; (A) Schematic depicting tRNA ${ }^{\text {Leu }}{ }_{\text {CAA }}$ (LeuX) and tRNA ${ }^{\text {Leu }}{ }_{\text {UAA }}$ (LeuZ) anticodons and their cognate mRNA codons (UUG or UUA). This schematic is modified from a leucine tRNA schematic obtained from the following source [20]; (B) tRNA ${ }^{\text {Leu }}{ }_{\text {CAA }}$ (LeuX) and tRNA ${ }^{\text {Leu }}{ }_{\text {UAA }}$ (LeuZ) secondary structure secondary structure. Nucleotides subject to modification are shown in grey and the sites of the MiaA, TrmL, and TusA modifications studied here are shown. This schematic is modified from Figure 3C of [21].

A major role for tRNA modifications is the promotion of translational fidelity, through suppression of frameshifting and ribosome stalling [22-24]. Modifications also improve reading frame maintenance in response to rare or underutilized codons [25,26]. In this work, we investigated the role of several tRNA modifications on rpoS or $h f q$ expression: $2^{\prime}-O$-methylation of Cytidine or Uridine, at the wobble position $(\mathrm{C} / \mathrm{Um})$, 2-thiouridine at the wobble position $\left(\mathrm{s}^{2} \mathrm{U}\right)$, as well as isopentyl adenosine $37\left(i^{6} \mathrm{~A}\right)$ (Figure 1).

C/U34m is catalyzed by the tRNA methyl transferase enzyme TrmL enzyme in E. coli $[21,27,28]$. The C/U34m modification occurs in leucine $\mathrm{tRNA}_{\mathrm{CmAA}}$ and $\mathrm{tRNA}_{\mathrm{cmnm} 5 \mathrm{UmAA}}$ (Figure 1) [21]. Both of these leucine tRNA isoacceptors recognize UUA-Leu and UUG-Leu codons, and also carry the $\mathrm{i}^{6} \mathrm{~A} 37$ tRNA modification that assists in the optimal decoding of the Leu codons during rpoS translation (Figure 1) [19]. The TrmL-catalyzed C/U34m modification of these leucine tRNAs isoacceptors requires 
$i^{6} \mathrm{~A} 37$ modification [21]. Taken together, this suggests that mia $A$ and $t r m L$ may work together to affect rpoS expression and we investigated the role the trmL in rpoS translation in this work.

The 2-thiolation $\left(\mathrm{s}^{2}\right)$ tRNA modification occurs in the anticodon stem loop (ASL) on uridines at the wobble position 34 (Figure 1) [29]. The 2-thiouridine ( $\left.s^{2} \mathrm{U} 34\right)$ wobble tRNA modification, like other tRNA modifications within the ASL, improves translational fidelity by suppressing translational frameshifting, promoting tight codon-anticodon interactions for tRNA ${ }^{\text {Gln }}$ UUG with CAA and CAG glutamine codons [30]. tRNA 2-thiouridine synthesizing protein A (TusA) is necessary for the synthesis of the $\mathrm{s}^{2} \mathrm{U} 34$ modification [31]. TusA point mutations were identified in a genetic screen for mutations affecting rpoS expression, decreasing $\sigma^{S}$ levels at the level of stability [32]. In addition, the $\mathrm{s}^{2} \mathrm{U} 34$ modification is also a component of a more complex modification present on C/U34m modified leucine tRNA isoacceptors, one of which is implicated in rpoS translation [19].

Host Factor for phage $\mathrm{Q} \beta(\mathrm{Hfq})$ is a co-factor necessary for the vast majority of trans-acting small regulatory RNAs in E. coli and other bacteria, and has many pleotropic roles in the cell [33,34]. It was first discovered as a host factor for Bacteriophage $\mathrm{Q} \beta$ replication [35]. The $h f q$ gene is in a complex operon with multiple promoters and is immediately downstream of the $i^{6} \mathrm{~A} 37$ prenyl transferase miaA [36]. There is an elevated UUX-Leu to CUX-Leu ratio in the $h f q$ open reading frame, characterizing it as a HULC protein that may, like RpoS, be sensitive to the $i^{6} \mathrm{~A} 37$ tRNA modification [19]. There is little known about the translational regulation of the $h f q \mathrm{ORF}$, in particular the contribution of tRNA modifications.

Here we used a genetic approach to test the role of the TrmL-catalyzed and TusA-catalyzed tRNA modifications during rpoS expression. We also further tested our previous predictions on the role of the $i^{6} \mathrm{~A} 37$ modification on expression of proteins with High UUX leucine Codon (HULC) content, using $h f q$ as a model gene. Hfq was an attractive candidate, due to its elevated UUX-Leu codon usage ratio (Table S7, [19]) and its phylogenetically conserved cotranscription with miaA. Here we demonstrate that both TrmL and TusA are necessary for full RpoS translation and MiaA-catalyzed-i ${ }^{6} \mathrm{~A} 37$ is necessary for $h f q$ expression.

\section{Results}

\subsection{Wobble Base tRNA Modifications Enzymes TrmL and TusA Are Necessary for RpoS Expression}

In order to determine if $\mathrm{mnm}^{5} \mathrm{~s}^{2} \mathrm{U} 34$ and C/U34m tRNA modifications may play a role in facilitating proper rpoS translation, we measured the effect of mutations in the enzymes necessary for these modifications on rpoS expression, using two different rpoS-lacZ translational fusion strains. Here, we will assume that phenotypes associated with absence of the enzyme are due to lack of the modification, although it is possible these enzymes have other roles in the cell.

The first translational fusion strain contains the rpoS promoter, the $5^{\prime}$ untranslated region, and 750 nucleotides of the open reading frame (rpoS750-lacZ) fused, in frame, to the ninth codon of the lac $Z$ ORF. The second translational fusion strain has the arabinose inducible $\mathrm{P}_{B A D}$ promoter in place of the rpoS promoter and $5^{\prime}$ untranslated region (5' UTR), as well as the complete open reading frame, except the termination codon ( $\mathrm{P}_{B A D^{-}}$-rpoS990-lacZ) fused, in frame, to the ninth codon of the lacZ ORF. Both of these fusions were previously used to characterize the contribution of the MiaA-catalyzed $i^{6} \mathrm{~A} 37$ tRNA modification in rpoS translation $[15,19]$. We transduced zeomycin-linked null mutations in trmL ( $\Delta$ trmL::zeo) and tusA ( $\triangle$ tusA::zeo) into both the rpoS750-lacZ and $P_{B A D}$-rpoS990-lacZ translational fusion strains and measured $\beta$-galactosidase expression compared to the wild type control strain (Figures 1B and 2A). For the rpoS750-lacZ translational fusion-at Optical Density $600 \mathrm{~nm}\left(\mathrm{OD}_{600}\right)$ $0.5,1.0,1.5$, and 2.0 - the activity of the rpoS-lacZ fusion was virtually undetectable in the absence of $\operatorname{trmL}$ or $t u s A$ at $<1.0$ machine units (Figure 2A). Arabinose led to significant induction of the wild

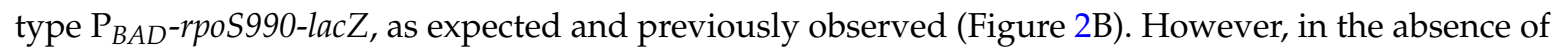
trmL or tusA this fusion also had virtually undetectable activity, with specific activities $<1.0$ machine units throughout the $30 \mathrm{~min}$ following induction (Figure 2B). Taken together, the decreased activity of 
the rpoS fusions in the absence of $t u s A$ and $t r m L$ suggest that the presence of the $s^{2} \mathrm{U} 34$ and C/U34m tRNA modifications are necessary for $r p o S$ expression. Any effects seen with both fusions cannot be attributed to the native promoter or the $5^{\prime}$ UTR (and thus sRNA regulation of RpoS), since neither the native promoter nor the $5^{\prime}$ UTR are present in the $P_{B A D}$ fusion. Effects on RpoS-LacZ levels could reflect differences in translation, in mRNA stability, or in protein degradation; these possibilities are explored in the discussion.

A

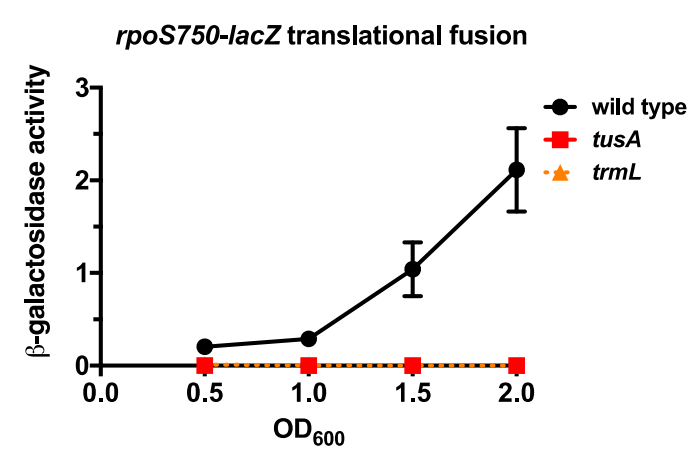

B

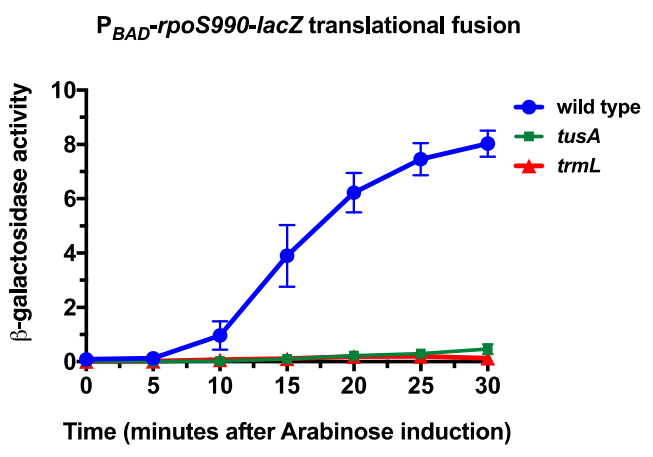

Figure 2. The effect of $s^{2} \mathrm{U} 34$ and $\mathrm{C} / \mathrm{U} 34 \mathrm{~m}$ tRNA modifications on rpoS-lacZ expression; (A) Wild type (EM1050), $\mathrm{rrmL}^{-}$(KMT767), and tus $A^{-}$(KMT766) rpoS750-lacZ translational fusion strains were grown in Luria Bertani (LB) Lennox media at $37^{\circ} \mathrm{C}$ and $200 \mathrm{rpm}$. Aliquots were taken at Optical Density $600 \mathrm{~nm}\left(\mathrm{OD}_{600}\right)$ of $0.5,1.0,1.5$, and 2.0 for $\beta$-galactosidase assay; (B) Wild type (KMT30003), trmL $L^{-}$(KMT30003), and tus $A^{-}$(KMT30003) $\mathrm{P}_{B A D^{-}}$rpoS990-lacZ translational fusion strains (in rssB ${ }^{-}$ backgrounds) were grown in LB media, supplemented with glucose to a final concentration of $0.2 \%$, at $37^{\circ} \mathrm{C}$ to an $\mathrm{OD}_{600}$ of 0.5 . Cells were harvested by centrifugation and resuspended in LB media, supplemented with arabinose to a final concentration of $0.2 \%$, and further incubated at $37^{\circ} \mathrm{C}$. Aliquots were taken at $5 \mathrm{~min}$ intervals for $30 \mathrm{~min}$ for $\beta$-galactosidase assay.

\subsection{TrmL tRNA Modification is Necessary for Decoding of UUX-Leucine Decoding in RpoS}

We previously demonstrated that the $i^{6} \mathrm{~A} 37$ tRNA modification was required for UUX-leu decoding, with silent UUX-Leu to CUX-Leu codon mutations in the rpoS reading frame partially suppressing the $i^{6} \mathrm{~A} 37$ requirement for expression [19]. In addition, since the C/U34m occurs in certain leucine $\mathrm{tRNAs}$, requires the MiaA-catalyzed $\mathrm{i}^{6} \mathrm{~A} 37 \mathrm{tRNA}$ modification, and may be necessary for $r p o S$ translation (Figure 1), we asked whether the trmL requirement for RpoS expression may be related to UUX-leucine decoding, using our previously described UUX to CUX mutant derivatives of the

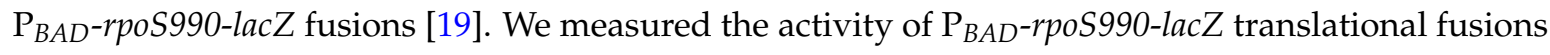
in which UUA-Leu codons with the rpoS portion of the fusion ORF have been changed to CUX-Leu $\left(l e u^{*} 1\right)$, UUG-Leu to CUX-Leu (leu*2), or UUA-Leu and UUG-Leu to CUX-Leu (leu*3) mutations, in the presence and absence of $\operatorname{trmL}$ (Figure 3). Either the UUA-Leu to CUX-Leu codon construct (leu*1) or the UUG-Leu to CUX-Leu construct (leu*2) in the rpoS ORF completely suppressed the trmL requirement for rpoS expression (Figures 2B and 3A). Finally, combined UUA-Leu to CUX-Leu and UUG-Leu to CUX-Leu codon mutations within the rpoS ORF also completely suppressed the trmL requirement (Figure 3C). Taken together, this strongly supports the idea that the TrmL-catalyzed C/U34m tRNA modification is necessary for rpoS translation, dependent upon the presence of UUX-Leu codons. 
A

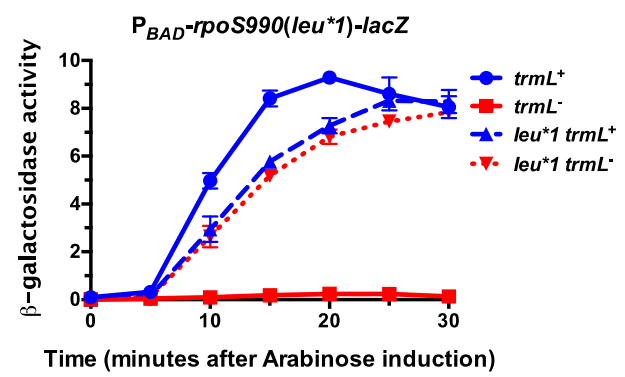

C

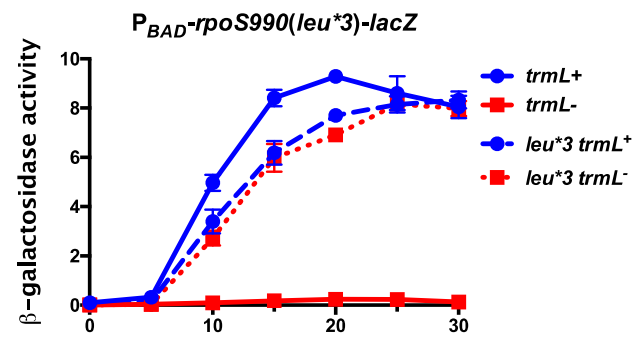

B

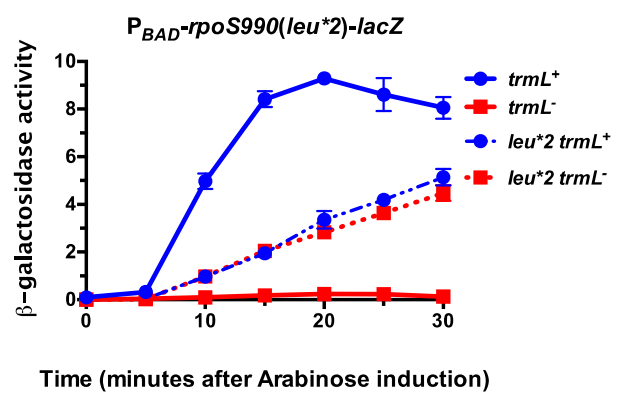

Time (minutes after Arabinose induction)

Figure 3. trmL is necessary for leucine decoding during RpoS expression. $t r m L^{+}$and $t r m L^{-}$ $\mathrm{P}_{B A D^{-}}$rpoS990-lacZ translational fusions (in $r s s B^{-}$backgrounds) were grown in LB, supplemented with glucose to a final concentration of $0.2 \%$, at $37{ }^{\circ} \mathrm{C}$ to an $\mathrm{OD}_{600}$ of 0.5 . Cells were harvested by centrifugation and res-suspended in LB supplemented with arabinose to a final concentration of $0.2 \%$. Finally, $100 \mathrm{~mL}$ aliquots of culture were isolated at 5 -min intervals for $30 \mathrm{~min}$. This process was executed for $\mathrm{P}_{B A D}$-rpoS990-lacZ translational fusions with rpoS UUA-Leu CUX-Leu mutations (leu*1)-strains KMT36002 and KMT36010 (A); rpoS UUG-Leu to CUX-leu mutation (leu*2)-strains KMT37002 and KMT37010 (B); rpoS with both UUA-Leu to CUX-Leu and UUG-Leu to CUX-Leu mutations (leu*3)-strains KMT33001 and KMT33013 (C). All time points represent the average of three independent experiments (biological replicates) and the error bars represent the standard error of the mean.

\subsection{MiaA Is Necessary, While TrmL Is Dispensable, for Hfq Expression}

We previously identified the UUX-Leu ratios of all open reading frames within the E. coli genome and proposed using this ratio as a predictor of $i^{6} \mathrm{~A} 37$ sensitivity during translation of the open reading frame [19]. We measured the effect of mutations in miaA on expression of another gene known to be involved in the RpoS regulatory circuitry, that also has a UUX-Leu ratio suggestive of $i^{6} \mathrm{~A} 37$ sensitivity, the RNA-chaperone Hfq. We hypothesized that additional HULC proteins may be sensitive to the presence of the $\mathrm{C} / \mathrm{U} 34 \mathrm{~m}$ modification, since the expression of at least two predicted HULC were defective in the miaA mutant and the C/U34m modification requires the MiaA-catalyzed $i^{6}$ A37 modification. An arabinose inducible $h f q$ fusion containing the $h f q$ open reading frame, except the termination codon, was fused in-frame, with the ninth codon of the lacZ ORF ( $\mathrm{P}_{\mathrm{BAD}}$-hfq306-lacZ-Figure 4A). Next, we transduced in zeomycin-linked null mutations in trmL $(\Delta t r m L:: z e o)$ and miaA ( $\Delta$ miaA::zeo), and measured $\mathrm{P}_{\mathrm{BAD}}$-hfq306-lacZ activity in the wild-type, trmL, and miaA mutants following arabinose induction. The activity of the wild-type fusion increased in a time-dependent manner following arabinose induction (Figure 4). The trmL mutation had no difference in the activity of the fusion following induction. However, there were some differences in the activity of the $h f q$ fusion in the absence of the absence of the MiaA-catalyzed i ${ }^{6}$ A37 tRNA modification. Overall, 
expression of the fusion was slower to increase in the miaA mutant mutant, with significantly lower levels most obvious at 10 and $15^{\prime}$ (Figure 4 and Table 1).

Taken together, this suggests that while the TrmL-catalyzed C/U34m tRNA modification is dispensable for $h f q$ translation, the MiaA catalyzed $i^{6} \mathrm{~A} 37$ is necessary for efficient $h f q$ translation. This also provides experimental evidence that a $3^{\text {rd }}$ HULC protein, in addition to rpoS and iraP, requires the $\mathrm{i}^{6} \mathrm{~A} 37 \mathrm{tRNA}$ modification, although further confirmation of a direct effect of the miaA mutant on $h f q$ translation will require testing a version of the $h f q$ fusion in which the UUX codons have been changed to CUX codons.

A

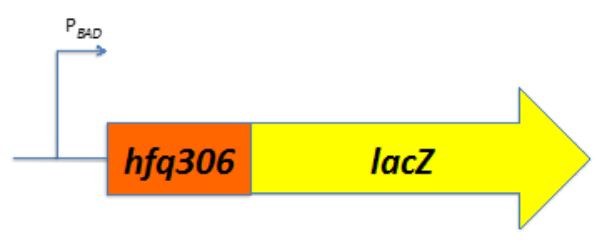

B

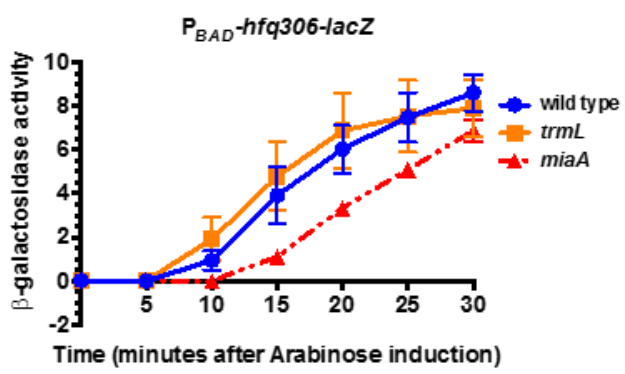

Figure 4. miaA, not trmL, is necessary for full $h f q$ expression. (A) Schematic depicting the $\mathrm{P}_{B A D}$-hfq306-lacZ translational fusion used for this experiment. (B) miaA $^{+}{ }^{t r m L} L^{+}$(KMT38000), miaA ${ }^{+}$ trmL-(KMT38004), and miaA $A^{-}$trmL $^{+}$(KMT38002) $\mathrm{P}_{B A D}-$ hfq306-lacZ translational fusion strains were grown in LB media, supplemented with glucose to a final concentration of $0.2 \%$ at $37^{\circ} \mathrm{C}$ to an $\mathrm{OD}_{600}$ of 0.5. Cells were harvested by centrifugation and resuspended in LB media, supplemented with arabinose to a final concentration of $0.2 \%$, and further incubated at $37^{\circ} \mathrm{C}$. Aliquots were taken at $5 \mathrm{~min}$ intervals for $30 \mathrm{~min}$ for $\beta$-galactosidase assay. All time points represent the average of three independent experiments (biological replicates) and the error bars represent the standard error of the mean.

Table 1. $\mathrm{P}_{\mathrm{BAD}}-h f q 306-l a c Z$ translational fusion $m i a A^{+} / m_{i a A^{-}}$activity ratio.

\begin{tabular}{|c|c|c|c|}
\hline \multirow{2}{*}{$\begin{array}{l}\text { Time (Min after Ara } \\
\text { Induction) }\end{array}$} & \multicolumn{3}{|c|}{ Strain (Mean $\beta$-Gal Activity) } \\
\hline & $h f q^{+}$ & $h f q^{-}$ & $h f q^{+} / h f q^{-}$Fold Change \\
\hline 10 & 0.94 & 0.00 & $\infty$ \\
\hline 15 & 3.91 & 1.11 & 3.52 \\
\hline 20 & 6.04 & 3.35 & 1.80 \\
\hline 25 & 7.50 & 5.10 & 1.47 \\
\hline 30 & 8.62 & 6.89 & 1.25 \\
\hline
\end{tabular}

\section{Discussion}

\subsection{Expanded Network of tRNA Modifications Affecting rpoS Expression and Physiological Implications}

Prior to this work, there was only one report of a tRNA modification, $\mathrm{i}^{6} \mathrm{~A} 37$, directly influencing rpoS translation [15,19]. Since there are multiple post-transcriptional regulators of RpoS, we hypothesized that additional tRNA modifications may be necessary for efficient translation of RpoS. Here we examined two additional tRNA modifications as possible regulators of $r p o S$ expression, TusA-catalyzed s² $\mathrm{U}$ and TrmL-catalyzed C/Um.

Both of these tRNA modifications occur at the wobble position and are likely to influence rpoS expression through improving proper codon-anticodon interactions at the wobble position where non-canonical RNA-RNA interactions can occur. The C/Um modification occurs on leucine tRNA

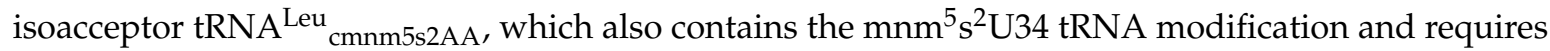
the $\mathrm{ms}^{2} \mathrm{i}^{6} \mathrm{~A} 37 \mathrm{tRNA}$ modification [21]. The TusA catalyzed $\mathrm{s}^{2} \mathrm{U} 34$ modification is also a precursor 
for the 5-carboxymethylaminomethyl-2-thiouridine ( $\left.\mathrm{cmnm}^{5} \mathrm{~s}^{2} \mathrm{U} 34\right)$ tRNA hypermodification or the 5-methylaminomethyl-2-thiouridine ( $\left.\mathrm{mnm}^{5} \mathrm{~s}^{2} \mathrm{U} 34\right)$ tRNA modification via the Methylaminomethyl modification G/E (MnmG/E) pathway [37,38].

Our previous experiments suggested that the requirement for MiaA (assumed in this discussion to reflect a requirement for the $i^{6} \mathrm{~A} 37$ modification) was due to direct effects on decoding of rpoS. That evidence started from the observation that rpoS, unlike rpoD, was enriched for UUX leucine codons (termed here HULC for High UUX-leucine codon) $[15,19]$. The tRNA, tRNA ${ }^{\text {Leu }}$ CAA (encoded by leuX) that is the target for these modifications, acts as a multi-copy suppressor of the $i^{6} \mathrm{~A} 37$ requirement for optimal rpoS expression [19], consistent with UUX leucine codons limiting translation. Finally, rpoS codon swapping experiments, specifically changing UUX-Leu to CUX-Leu, demonstrated partial suppression of the MiaA requirement during rpoS expression [19], ruling out more indirect effects on translation. The presence of the TrmL-catalyzed C/Um modification on the tRNA ${ }^{\text {Leu }}{ }_{C A A}$ isoacceptor and the necessity of both TrmL and MiaA for complete rpoS translation suggest that TrmL and MiaA-catalyzed tRNA modifications could work together to optimize $r p o S$ translation. This was tested here; the trmL requirement for rpoS translation in our experimental model were much more dramatic than the miaA requirement for rpoS expression for reasons that are not yet clear. However, because the trmL defect was fully suppressed when UUX codons in rpoS were changed to CUX codons (Figure 2), indirect effects of the trmL mutant on RpoS expression can be ruled out.

The precise physiological implications of the TrmL and MiaA effects on rpoS translation are still under investigation. However, previous reports offer some clues as to how these modifications have a more global impact on the cell. In long-term survival experiments, trmL mutants were less competitive than wild type cells, suggesting a role for trmL in stationary phase recovery [21]. That report is consistent with an important role for trmL in rpoS expression, since rpoS is necessary for stationary phase stress responses. In $E$. coli, the $\mathrm{mia}_{\mathrm{P} 3(\mathrm{HS})}$ transcript is elevated under extreme heat shock, $50{ }^{\circ} \mathrm{C}$ [36]. In Salmonella typhimurium, miaA mutants lack the ability to survive at $42{ }^{\circ} \mathrm{C}$ and are sensitive to oxidative stress [39]. These reports both suggest that MiaA levels are important during heat shock. Leucine supplementation or suppression of the leu operon were able to suppress the sensitivity of miaA mutants to heat shock and oxidative stress [39]. Therefore, heat shock and/or leucine starvation may be critical conditions under which the $i^{6} \mathrm{~A} 37, \mathrm{~s}^{2} \mathrm{U} 34$, and $\mathrm{C} / \mathrm{Um}$ are necessary for $r p o S$ translation. Heat shock could increase the translation requirement for leucine amino acids due to global protein denaturation. Under these limiting leucine conditions, proper incorporation of leucine tRNAs into the less commonly utilized UUX-Leu codons would be critical.

\subsection{TusA Catalyzed $s^{2} U 34$ and rpoS Translation}

The $\mathrm{s}^{2} \mathrm{U}$ requirement for rpoS expression, at the level of translation, adds to the current knowledge of the role that TusA plays on rpoS expression. Mutations in tusA were previously shown to decrease rpoS expression at the level of protein stability [32]. In this study, our rpoS translational fusions were in cells containing a deletion of the adaptor protein, RssB, which targets RpoS for degradation by the ClpXP protease; RpoS is stable in this strain background. Therefore, our observations suggest that tus $A$ is necessary for optimal translation as well. Taken together, it would appear that TusA decreases RpoS expression at the level of stability and translation. It is also possible that defective rpoS translation in the absence of trmL leads to increased RpoS degradation. Similar to the trmL effect on the rpoS fusion, the tusA effect on the rpoS fusion was much more dramatic than the mia $A$ effect for reasons that are not clear.

\subsection{The $i^{6}$ A37 Requirement for Hfq and Implications for HULC Protein Predictive Model and Small RNA Biology}

Hfq's critical role in the action of bacterial small regulatory RNAs makes it a pleotropic effector of cellular physiology [34,40]. In this work, we examine the role of tRNA modifications in $h f q$ translation at the level of the reading frame. We observed a two-fold decrease in Hfq levels in the absence of 
miaA (Figure 4), suggesting a role for $\mathrm{i}^{6} \mathrm{~A} 37 \mathrm{tRNA}$ modification during $h f q$ translation. Since Hfq levels are affected by the presence of $i^{6} A 37$, and sRNA steady state levels are affected by Hfq levels, we hypothesize that the presence of the $i^{6} \mathrm{~A} 37$ tRNA modification may be indirectly implicated in sRNA steady state levels.

Transcriptional regulation of $h f q$ has been more extensively characterized than post-transcriptional regulation of $h f q$ [41]. There are several $h f q$ promoters within the miaA gene, each with different activity levels; these include promoters for vegetative growth and heat shock $[36,41]$. The transcripts from this superoperon appear to undergo post-transcriptional regulation in an RNaseE dependent manner [41]. Based on these transcriptional mapping studies, $h f q$ should contain more than one $5^{\prime}$ untranslated region $[36,41]$. Proteins or small regulatory RNAs could target these $5^{\prime}$ UTRs for post-transcriptional regulation. The further examination of the $h f q$ reading frame translation will also contribute additional understanding of $h f q$ regulation and small RNA action. This is the first report on the role of tRNA modifications in Hfq expression.

While the identification of $h f q$ as a HULC protein suggested that it may be sensitive to the presence of the MiaA, this has not previously been tested [19]. In addition, there have only been a few predicted HULC proteins tested for MiaA sensitivity [19]. Demonstrating the $i^{6} \mathrm{~A} 37$ sensitivity for an additional HULC protein, $h f q$, strengthens the predictive power of this model [19]. Interestingly, unlike rpoS, trmL mutants had no effect on $h f q$ translation, suggesting that not all miaA effects are due to inability to add the TrmL-dependent modification (Figures 1 and 3). Based on our results (Figure 4), small regulatory RNA levels and activity may be influenced by $i^{6} \mathrm{~A} 37 \mathrm{tRNA}$ modification levels. Hfq is limiting under some conditions [42]. Therefore, decreased levels of Hfq under conditions that may decrease the $\mathrm{i}^{6} \mathrm{~A} 37$ tRNA modification, may limit small regulatory RNA levels and activity.

\subsection{Implications for the Prokaryotic and Eukaryotic Organisms}

In eukaryotic cells, tRNA modifications have been shown to play a regulatory role in cellular physiology and stress responses $[25,26,43-47]$. This includes cell cycle progression and metabolic deficiencies [25,47]. The stress responses that require tRNA modifications include global translational stress, oxidative stress, and DNA damage $[25,43,44]$. Since RpoS is considered a general stress adaptor for E. coli and other bacteria, the role of tRNA modifications in modulating its expression draws some parallels. Mycobacterial survival during hypoxia was recently shown to require tRNA modifications [48]. This suggests a broad role for tRNA modifications in allowing cells to respond to oxidative stress across the biological domains. Mitochondrial tRNA modifications are critical for the prevention of the rare genetic and neurodegenerative disorder Mitochondrial, Encephalopathy, Lactic acidosis, and Stroke (MELAS) syndrome [49]. Those with this disease lack the 5-taurinomethyl-2-thiouridine $\left(\tau \mathrm{m}^{5} \mathrm{~s}^{2}\right)$ on the wobble position of tRNA ${ }^{\text {LeuUUR }}$ [49]. Furthermore, miRNA-9/9* expression results in the post-transcriptional repression of several tRNA modification enzymes [49]. As a parallel, our observations with Hfq suggest bacterial small RNA involvement in tRNA modification biology. Interestingly, the tRNA modification in MELAS syndrome is associated with leucine decoding.

\section{Materials and Methods}

\subsection{Strains and Oligonucleotide Primers}

All strains were derivatives of Escherichia coli K12 MG1655. All strains used in this study are listed in Table S1. All oligonucleotide primers are used in this study are listed in Table S2.

\subsection{Growth Conditions and Media}

All bacteria were grown in Luria Bertani (LB) Media unless otherwise stated. In experiments requiring rapid induction of arabinose inducible fusions, LB was supplemented with $0.2 \%$ Glucose $(w / v)$ to repress the fusion, or $0.2 \%$ Arabinose $(w / v)$ to induce it. LB agar plates supplemented with 
zeomycin to a final concentration of $25 \mu \mathrm{g} / \mathrm{mL}$ were used to select for $\Delta$ tus $A:: z e o$ and $\Delta$ trmL::zeo recombinants or transductants. M63 minimal media supplemented with glycerol, 5\% sucrose, and $80 \mu \mathrm{g} / \mathrm{mL}$ of Xgal (M63-Gly-Suc-XG) agar plates were used for the positive selection of $\mathrm{P}_{B A D}$-hfq306-lacZ.

\subsection{Genetic Constructions}

The $\Delta t u s A:: z e o$ and $\Delta t r m L:: z e o$ mutations were created in DJ480 mini- $\lambda:$ tet via recombineering [50] and transduced into wild type or leu* versions of rpoS750-lacZ, $\mathrm{P}_{B A D}$-rpoS990-lacZ, $\mathrm{P}_{B A D}$-hfq306-lacZ translational fusions using Bacteriophage P1. Briefly, allelic exchange substrates for $\triangle t u s A:: z e o$ and $\Delta t r m L:: z e o$ mutations were created by PCR amplification of zeomycin resistance cassette using oligonucleotide primers KT1188 and KT1189 (Table S2) as well as KT1192 and KT1193 (Table S2), containing 40 nucleotides of flanking homology to tus $A$ and $t r m L$, respectively. The PCR products were analyzed by agarose gel electrophoresis and purified. An overnight culture of DJ480 mini- $\lambda$ ::tet was resuspended in fresh $\mathrm{LB}$ and grown at $30^{\circ} \mathrm{C}$ to an $\mathrm{OD}_{600}$ of 0.5 . The culture was then shifted to $43.5^{\circ} \mathrm{C}$ for $15 \mathrm{~min}$. Finally, these induced cells were washed with ice-cold $\mathrm{H}_{2} \mathrm{O}$ and resuspended in ice-cold $10 \%$ glycerol. Approximately $100 \mathrm{ng}$ of purified PCR products, corresponding to $\triangle$ tus $A:: z e o$ and $\Delta t r m L:: z e o$ allelic exchange substrates, were electroporated into induced DJ480 mini- $\lambda:: t e t$ cells, as well as a no DNA control, using $0.1 \mathrm{~mm}$ cuvettes and an electroporator at setting Ec1 (Biorad). Cells were recovered in $1 \mathrm{~mL}$ of LB for 30-60 min and a $100 \mu \mathrm{L}$ aliquot of cells were spread on LB agar plates supplemented with zeomycin to a final concentration of $25 \mu \mathrm{g} / \mathrm{mL}$ (LB-zeo). Zeomycin resistant recombinants or transductants were purified once on LB-zeo plates and twice on LB plates.

$\mathrm{P}_{B A D}-h f q 306-l a c Z$ translational fusions were constructed using PM1800 as previously described [19]. Briefly, allelic exchange substrates corresponding to $P_{B A D}-h f q 306-l a c Z$ were PCR amplified using synthetic gBlock DNA (IDT DNA) as a template and specific oligonucleotide primers (KT1160 and KT1161). The PCR products were analyzed by agarose gel electrophoresis and purified. An overnight culture of PM1800 was resuspended in fresh LB and grown at $30{ }^{\circ} \mathrm{C}$ to an $\mathrm{OD}_{600}$ of 0.5. The culture was then shifted to $43.5{ }^{\circ} \mathrm{C}$ for $15 \mathrm{~min}$. Finally, these induced cells were washed with ice-cold $\mathrm{H}_{2} \mathrm{O}$ and resuspended in ice-cold 10\% glycerol. Approximately $100 \mathrm{ng}$ of purified PCR products, corresponding to $\mathrm{P}_{B A D}$-hfq306-lacZ allelic exchange substrates were electroporated into induced PM1800 cells, as well as a no DNA control, using $0.1 \mathrm{~mm}$ cuvettes and an electroporator at setting Ec1 (Biorad). Cells were recovered in $10 \mathrm{~mL}$ of LB for $18 \mathrm{~h}$. Recovered cultures were serially diluted down to $10^{-6}$ and $100 \mu \mathrm{L}$ aliquot of serial dilutions were spread on M63-Gly-Suc-XG. Colonies that were blue on XG were purified once on M63-Gly-Suc-XG plates once and twice on LB. Then, they were screened for chloramphenicol sensitivity and confirmed by PCR.

\section{4. $\beta$-Galactosidase Assays}

High-throughput kinetic $\beta$-galactosidase assays were carried out in 96-well plates as previously described [19]. The Filtermax F5 (Molecular Devices, Sunnyvale, CA 94089 USA) multimode microplate reader was used to read microtiter plates. $\beta$-galactosidase specific activity units are defined as the slope of $\mathrm{OD}_{420}$ reading divided by $\mathrm{OD}_{600}$ and are approximately 25-fold lower than Miller Units.

Experimental design for assays executed following arabinose induction. Briefly, samples to be assayed were grown in $5 \mathrm{~mL}$ of LB-Glu overnight at $37^{\circ} \mathrm{C}$ in a roller drum. Overnight cultures were diluted 1:1000 in $30 \mathrm{~mL}$ of fresh LB-Glu in a 125-mL Erlenmeyer flask and grown at $37^{\circ} \mathrm{C}$ in a shaking water bath at $200 \mathrm{rpm}$. When cultures reached an $\mathrm{OD}_{600}$ of 1.0, cells were harvested by centrifugation, and resuspended in $30 \mathrm{~mL}$ of fresh LB supplemented $0.2 \%$ arabinose and $100 \mu \mathrm{L}$ aliquots of each culture were taken every $5 \mathrm{~min}$ for $\beta$-galactosidase assays. Samples were collected in triplicate for each individual experiment and averages were taken as a representative sample for each experiment. The data presented represent the mean and standard error of the mean of at least three independent replicates. 


\section{Conclusions}

TrmL catalyzed tRNA methylation C/U34m and TusA catalyzed tRNA thiouridinylation are critical for rpoS expression in E. coli. Removal of UUX-codons from the rpoS open reading frame suppresses the rpoS requirement for trmL during translation. The MiaA catalyzed i ${ }^{6} \mathrm{~A} 37$ tRNA modification is required for full $h f q$ translation in E. coli.

Supplementary Materials: The following are available online at http://www.mdpi.com/2218-273X/7/2/39/s1, Table S1: Strain list, Table S2: Oligonucleotide list.

Acknowledgments: We would like to thank Susan Gottesman for her comments on this manuscript. We would like to thank Bev Victor/Africa on Paper for assisting with the creation of the tRNA schematics in Figure 1. This research was supported by the National Institute of General Medical Sciences of the National Institutes of Health under award number SC2 GM105419 (K.M.T.). This research was also supported by the National Science Foundation under the LSAMP program (HRD-1000286 - supporting M.O.). Any opinions, findings, and conclusions or recommendations expressed in this material are those of the author(s) and do not necessarily reflect the views of the National Institute of Health or the National Science Foundation.

Author Contributions: K.M.T. conceived and designed the experiments; J.I.A. and M.O. performed the experiments; K.M.T. analyzed the data; K.M.T. wrote the paper.

Conflicts of Interest: The founding sponsors had no role in the design of the study; in the collection, analyses, or interpretation of data; in the writing of the manuscript, or in the decision to publish the results.

\section{References}

1. Tanaka, K.; Takayanagi, Y.; Fujita, N.; Ishihama, A.; Takahashi, H. Heterogeneity of the principal $\sigma$ factor in Escherichia coli: The rpoS gene product, $\sigma^{38}$, is a second principal $\sigma$ factor of RNA polymerase in stationary-phase Escherichia coli. Proc. Natl. Acad. Sci. USA 1993, 90, 8303. [CrossRef] [PubMed]

2. Hengge-Aronis, R. Survival of hunger and stress: The role of rpoS in early stationary phase gene regulation in E. coli. Cell 1993, 72, 165-168. [CrossRef]

3. Sledjeski, D.D.; Gupta, A.; Gottesman, S. The small RNA, DsrA, is essential for the low temperature expression of rpoS during exponential growth in Escherichia coli. EMBO J. 1996, 15, 3993-4000. [PubMed]

4. Zhang, A.; Altuvia, S.; Tiwari, A.; Argaman, L.; Hengge-Aronis, R.; Storz, G. The OxyS regulatory RNA represses rpoS translation and binds the Hfq (HF-I) protein. EMBO J. 1998, 17, 6061-6068. [CrossRef] [PubMed]

5. Majdalani, N.; Cunning, C.; Sledjeski, D.D.; Elliott, T.; Gottesman, S. Dsra RNA regulates translation of rpoS message by an anti-antisense mechanism, independent of its action as an antisilencer of transcription. Proc. Natl. Acad. Sci. USA 1998, 95, 12462-12467. [CrossRef] [PubMed]

6. Majdalani, N.; Hernandez, D.; Gottesman, S. Regulation and mode of action of the second small RNA activator of rpoS translation, RprA. Mol. Microbiol. 2002, 46, 813-826. [CrossRef] [PubMed]

7. Majdalani, N.; Chen, S.; Murrow, J.; St. John, K.; Gottesman, S. Regulation of rpoS by a novel small RNA: The characterization of RprA. Mol. Microbiol. 2001, 39, 1382-1394. [CrossRef] [PubMed]

8. Mandin, P.; Gottesman, S. Integrating anaerobic/aerobic sensing and the general stress response through the ArcZ small RNA. EMBO J. 2010, 29, 3094-3107. [CrossRef] [PubMed]

9. Zhou, Y.; Gottesman, S. Regulation of proteolysis of the stationary-phase $\sigma$ factor rpoS. J. Bacteriol. 1998, 180, 1154-1158. [PubMed]

10. Bougdour, A.; Wickner, S.; Gottesman, S. Modulating RssB activity: IraP, a novel regulator of $\sigma^{\mathrm{s}}$ stability in Escherichia coli. Genes Dev. 2006, 20, 884-897. [CrossRef] [PubMed]

11. Battesti, A.; Tsegaye, Y.M.; Packer, D.G.; Majdalani, N.; Gottesman, S. H-NS regulation of IraD and IraM antiadaptors for control of rpos degradation. J. Bacteriol. 2012, 194, 2470-2478. [CrossRef] [PubMed]

12. Bougdour, A.; Cunning, C.; Baptiste, P.J.; Elliott, T.; Gottesman, S. Multiple pathways for regulation of sigmas (rpoS) stability in Escherichia coli via the action of multiple anti-adaptors. Mol. Microbiol. 2008, 68, $298-313$. [CrossRef] [PubMed]

13. Battesti, A.; Majdalani, N.; Gottesman, S. Stress $\sigma$ factor rposS degradation and translation are sensitive to the state of central metabolism. Proc. Natl. Acad. Sci. USA 2015, 112, 5159-5164. [CrossRef] [PubMed] 
14. Gottesman, S.; Roche, E.; Zhou, Y.; Sauer, R.T. The ClpXP and ClpaAP proteases degrade proteins with carboxy-terminal peptide tails added by the SsrA-tagging system. Genes Dev. 1998, 12, 1338-1347. [CrossRef] [PubMed]

15. Thompson, K.M.; Gottesman, S. The MiaA tRNA modification enzyme is necessary for robust rpoS expression in Escherichia coli. J. Bacteriol. 2014, 196, 754-761. [CrossRef] [PubMed]

16. Caillet, J.; Droogmans, L. Molecular cloning of the Escherichia coli miaA gene involved in the formation of delta 2-isopentyl adenosine in tRNA. J. Bacteriol. 1988, 170, 4147-4152. [CrossRef] [PubMed]

17. Connolly, D.M.; Winkler, M.E. Structure of Escherichia coli K-12 miaa and characterization of the mutator phenotype caused by miaa insertion mutations. J. Bacteriol. 1991, 173, 1711-1721. [CrossRef] [PubMed]

18. Connolly, D.M.; Winkler, M.E. Genetic and physiological relationships among the miaA gene, 2-methylthio-N6-(delta 2-isopentenyl)-adenosine tRNA modification, and spontaneous mutagenesis in Escherichia coli K-12. J. Bacteriol. 1989, 171, 3233-3246. [CrossRef] [PubMed]

19. Aubee, J.I.; Olu, M.; Thompson, K.M. The i6a37 tRNA modification is essential for proper decoding of UUX-Leucine codons during rpoS and iraP translation. RNA 2016, 22, 729-742. [CrossRef] [PubMed]

20. Wobble. Available online: http://www.mun.ca/biology/scarr/iGen3_06-08.html (accessed on 24 April 2017).

21. Benitez-Paez, A.; Villarroya, M.; Douthwaite, S.; Gabaldon, T.; Armengod, M.E. Yibk is the 2-o-methyltransferase trml that modifies the wobble nucleotide in Escherichia coli tRNA(leu) isoacceptors. RNA 2010, 16, 2131-2143. [CrossRef] [PubMed]

22. Klassen, R.; Bruch, A.; Schaffrath, R. Independent suppression of ribosomal +1 frameshifts by different tRNA anticodon loop modifications. RNA Biol. 2016, 1-8. [CrossRef] [PubMed]

23. Tukenmez, H.; Xu, H.; Esberg, A.; Bystrom, A.S. The role of wobble uridine modifications in +1 translational frameshifting in eukaryotes. Nucleic Acids Res. 2015, 43, 9489-9499. [CrossRef] [PubMed]

24. Urbonavicius, J.; Qian, Q.; Durand, J.M.; Hagervall, T.G.; Bjork, G.R. Improvement of reading frame maintenance is a common function for several tRNA modifications. EMBO J. 2001, 20, 4863-4873. [CrossRef] [PubMed]

25. Patil, A.; Dyavaiah, M.; Joseph, F.; Rooney, J.P.; Chan, C.T.; Dedon, P.C.; Begley, T.J. Increased tRNA modification and gene-specific codon usage regulate cell cycle progression during the DNA damage response. Cell Cycle 2012, 1, 3656-3665. [CrossRef] [PubMed]

26. Endres, L.; Dedon, P.C.; Begley, T.J. Codon-biased translation can be regulated by wobble-base tRNA modification systems during cellular stress responses. RNA Biol. 2015, 12, 603-614. [CrossRef] [PubMed]

27. Liu, R.J.; Zhou, M.; Fang, Z.P.; Wang, M.; Zhou, X.L.; Wang, E.D. The tRNA recognition mechanism of the minimalist SPOUT methyltransferase, TrmL. Nucleic Acids Res. 2013, 41, 7828-7842. [CrossRef] [PubMed]

28. Zhou, M.; Long, T.; Fang, Z.P.; Zhou, X.L.; Liu, R.J.; Wang, E.D. Identification of determinants for tRNA substrate recognition by Escherichia coli C/U34 2'-O-methyltransferase. RNA Biol. 2015, 12, 900-911. [CrossRef] [PubMed]

29. Rogers, K.C.; Crescenzo, A.T.; Soll, D. Aminoacylation of transfer RNAs with 2-thiouridine derivatives in the wobble position of the anticodon. Biochimie 1995, 77, 66-74. [CrossRef]

30. Rodriguez-Hernandez, A.; Spears, J.L.; Gaston, K.W.; Limbach, P.A.; Gamper, H.; Hou, Y.; Kaiser, R.; Agris, P.F.; Perona, J.J. Structural and mechanistic basis for enhanced translational efficiency by 2-thiouridine at the tRNA anticodon wobble position. J. Mol. Biol. 2013, 425, 3888-3906. [CrossRef] [PubMed]

31. Ikeuchi, Y.; Shigi, N.; Kato, J.; Nishimura, A.; Suzuki, T. Mechanistic insights into sulfur relay by multiple sulfur mediators involved in thiouridine biosynthesis at tRNA wobble positions. Mol. Cell 2006, 21, 97-108. [CrossRef] [PubMed]

32. Yamashino, T.; Isomura, M.; Ueguchi, C.; Mizuno, T. The yhhp gene encoding a small ubiquitous protein is fundamental for normal cell growth of Escherichia coli. J. Bacteriol. 1998, 180, 2257-2261. [PubMed]

33. Tsui, H.C.; Leung, H.C.; Winkler, M.E. Characterization of broadly pleiotropic phenotypes caused by an hfq insertion mutation in Escherichia coli K-12. Mol. Microbiol. 1994, 13, 35-49. [CrossRef] [PubMed]

34. De Lay, N.; Schu, D.J.; Gottesman, S. Bacterial small RNA-based negative regulation: Hfq and its accomplices. J. Biol. Chem. 2013, 288, 7996-8003. [CrossRef] [PubMed]

35. Kajitani, M.; Ishihama, A. Identification and sequence determination of the host factor gene for bacteriophage Q beta. Nucleic Acids Res. 1991, 19, 1063-1066. [CrossRef] [PubMed] 
36. Tsui, H.C.; Feng, G.; Winkler, M.E. Transcription of the mutL repair, miaA tRNA modification, $h f q$ pleiotropic regulator, and $h f l A$ region protease genes of Escherichia coli K-12 from clustered E $\sigma^{32}$-specific promoters during heat shock. J. Bacteriol. 1996, 178, 5719-5731. [CrossRef] [PubMed]

37. Armengod, M.E.; Meseguer, S.; Villarroya, M.; Prado, S.; Moukadiri, I.; Ruiz-Partida, R.; Garzon, M.J.; Navarro-Gonzalez, C.; Martinez-Zamora, A. Modification of the wobble uridine in bacterial and mitochondrial tRNAs reading NNA/NNG triplets of 2-codon boxes. RNA Biol. 2014, 11, 1495-1507. [CrossRef] [PubMed]

38. Armengod, M.E.; Moukadiri, I.; Prado, S.; Ruiz-Partida, R.; Benitez-Paez, A.; Villarroya, M.; Lomas, R.; Garzon, M.J.; Martinez-Zamora, A.; Meseguer, S.; et al. Enzymology of tRNA modification in the bacterial mnmeg pathway. Biochimie 2012, 94, 1510-1520. [CrossRef] [PubMed]

39. Blum, P.H. Reduced leu operon expression in a miaA mutant of Salmonella typhimurium. J. Bacteriol. 1988, 170, 5125-5133. [CrossRef] [PubMed]

40. Papenfort, K.; Vogel, J. Multiple target regulation by small noncoding RNAs rewires gene expression at the post-transcriptional level. Res Microbiol. 2009, 160, 278-287. [CrossRef] [PubMed]

41. Tsui, H.C.; Winkler, M.E. Transcriptional patterns of the mutl-miaA superoperon of Escherichia coli K-12 suggest a model for posttranscriptional regulation. Biochimie 1994, 76, 1168-1177. [CrossRef]

42. Moon, K.; Gottesman, S. Competition among hfq-binding small RNAs in Escherichia coli. Mol. Microbiol. 2011, 82, 1545-1562. [CrossRef] [PubMed]

43. Patil, A.; Chan, C.T.; Dyavaiah, M.; Rooney, J.P.; Dedon, P.C.; Begley, T.J. Translational infidelity-induced protein stress results from a deficiency in Trm9-catalyzed tRNA modifications. RNA Biol. 2012, 9, 990-1001. [CrossRef] [PubMed]

44. Chan, C.T.; Pang, Y.L.; Deng, W.; Babu, I.R.; Dyavaiah, M.; Begley, T.J.; Dedon, P.C. Reprogramming of tRNA modifications controls the oxidative stress response by codon-biased translation of proteins. Nat. Commun. 2012, 3, 937. [CrossRef] [PubMed]

45. Chan, C.T.; Dyavaiah, M.; DeMott, M.S.; Taghizadeh, K.; Dedon, P.C.; Begley, T.J. A quantitative system approach reveals dynamic control of tRNA modifications during cellular stress. PLoS Genet. 2010, 6, e1001247. [CrossRef] [PubMed]

46. Begley, T.J.; Dyavaiah, M.; Patil, A.; Rooney, J.P.; Direnzo, D.; Young, C.M.; Conklin, D.S.; Zitomer, R.S.; Begley, T.J. Trm-9 catalized tRNA modifications link translation to the DNA damage response. Mol. Cell 2007, 28, 860-870. [CrossRef] [PubMed]

47. Lamichhane, T.N.; Blewett, N.H.; Crawford, A.K.; Cherkasova, V.A.; Iben, J.R.; Begley, T.J.; Farabaugh, P.J.; Maraia, R.J. Lack of tRNA modification isopentenyl-a37 alters mRNA decoding and causes metabolic deficiences in fission yeast. Mol. Cell. Biol. 2013, 33, 2918-2929. [CrossRef] [PubMed]

48. Chionh, Y.H.; McBee, M.; Babu, I.R.; Hia, F.; Lin, W.; Zhao, W.; Cao, J.; Dziergowska, A.; Malkiewicz, A.; Begley, T.J.; et al. tRNA-mediated codon-biased translation in mycobacterial hypoxic persistence. Nat. Commun. 2016, 11, 13302. [CrossRef] [PubMed]

49. Meseguer, S.; Martinez-Zamora, A.; Garcia-Arumi, E.; Andreu, A.L.; Armengod, M.E. The ROS-sensitive microRNA-9/9* controls the expression of mitochondrial tRNA modifyng enzymes and is involved in the molecular mechanism of melas syndrome. Hum. Mol. Genet. 2015, 24, 167-184. [CrossRef] [PubMed]

50. Court, D.L.; Swaminathan, S.; Yu, D.; Wilson, H.; Baker, T.; Bubunenko, M.; Sawitzke, J.; Sharan, S.K. Mini- $\lambda$ : A tractable system for chromosome and bac engineering. Gene 2003, 315, 63-69. [CrossRef]

(C) 2017 by the authors. Licensee MDPI, Basel, Switzerland. This article is an open access article distributed under the terms and conditions of the Creative Commons Attribution (CC BY) license (http://creativecommons.org/licenses/by/4.0/). 